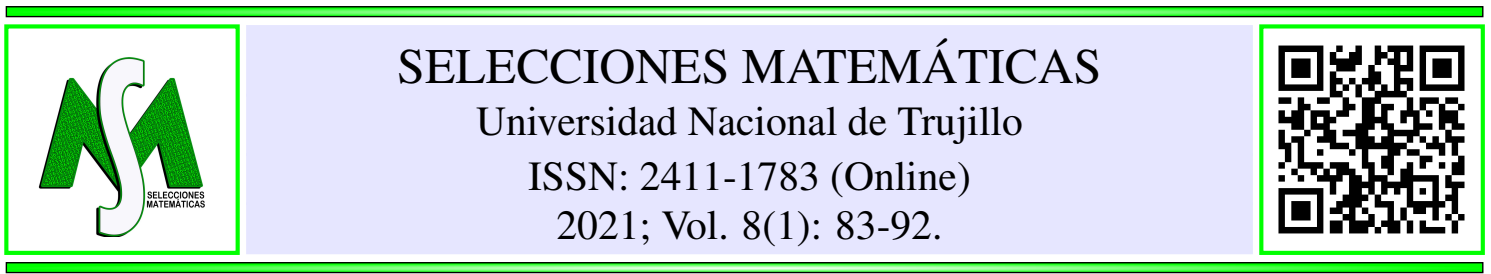

\title{
Hypersurfaces of the spherical type in Euclidean spaces
}

\author{
Carlos M. C. Riveros ${ }^{\circledR}$ and Cid D. F. Machado ${ }^{\circledR}$
}

Received, Jan. 26, 2021

Accepted, Jun. 30, 2021

How to cite this article:

Riveros Carlos, Machado Cid. Hypersurfaces of the spherical type in Euclidean spaces. Selecciones Matemáticas. 2021;8(1):83-92. http://dx.doi.org/10.17268/sel.mat.2021.01.08

\begin{abstract}
In this paper we study a class of oriented hypersurfaces in Euclidean space, namely, the hypersurfaces of the spherical type, this class of hypersurfaces includes the surfaces of the spherical type (Laguerre minimal surfaces) studied in [8]. We show that for $n=2$, the classes of surfaces of the spherical type and the Weingarten surfaces of the spherical type coincide, more for larger dimensions this is not true and we give explicit examples. We also introduced a class of hypersurfaces associated to a biharmonic map and we show that the hypersurfaces of the spherical type are associated to a biharmonic map. Moreover, we classify the hypersurfaces of the spherical type of rotation.
\end{abstract}

Keywords . Weingarten hypersurfaces of the spherical type, Laguerre minimal surfaces, biharmonic map, rth mean curvature.

1. Introduction. The surfaces $M^{2}$ satisfying a functional relation of the form $W(H, K)=0$, where $H$ and $K$ are the mean and Gaussian curvatures of the surface $M^{2}$, respectively, are called Weingarten surfaces. Examples of Weingarten surfaces are the surfaces of revolution and the surfaces of constant mean or Gaussian curvature. In [7], the authors study an important class of surfaces satisfying a linear relation of the form

$$
a H+b K+c=0,
$$

where $a, b, c \in \mathbb{R}$ and $a^{2}+b^{2} \neq 0$. These surfaces are called linear Weingarten surfaces.

In [5], Corro presented a way of parameterizing surfaces as envelopes of a congruence of spheres in which an envelope is contained in a plane and with radius function $h$ associated with a hydrodynamic type system. As an application, it studies the surfaces in hyperbolic space $\mathbb{H}^{3}$ satisfying the equality

$$
2 a c h \frac{2(c-1)}{c}(H-1)+\left(a+b-a c h \frac{2(c-1)}{c}\right) K=0,
$$

where $a, b, c \in \mathbb{R}, a+b \neq 0, c \neq 0, H$ is the mean curvature and $K$ is the Gaussian curvature. This class of surfaces includes the Bryant surfaces and the flat surfaces of the hyperbolic space and are called generalized Weingarten surfaces of Bryant type.

An oriented surface $\psi: M^{2} \rightarrow \mathbb{R}^{3}$ with non-zero Gaussian curvature $K$ and mean curvature $H$ is called a Laguerre minimal surface if

$$
\Delta_{I I I}\left(\frac{H}{K}\right)=0,
$$

where $\Delta_{I I I}$ is the Laplacian with respect to the third fundamental form $I I I$ of $\psi$. The study of these surfaces was done by W. Blaschke [1, 2, 3, 4], where such surfaces appear as critical points of the functional

$$
L(\Psi)=\int \frac{H^{2}-K}{K} d M,
$$

\footnotetext{
*Departamento de Matemática, Universidade de Brasília, 70910-900, Brasília-DF, Brazil. (car los @mat . unb.br).

${ }^{\dagger}$ FacUnicamps, CEP 74535-280, Goiânia-GO, Brazil. (ci dgeomat @gmai l . com).
} 
where $d M$ is the area element.

In [8], the authors study Laguerre's minimal surfaces as graphs of biharmonic functions in the isotropic model of Laguerre Geometry. In particular, they study the surfaces of the spherical type (Laguerre minimal surfaces), namely the surfaces $M^{2}$ of $\mathbb{R}^{3}$ such that the set of spheres with center $p+\frac{H(p)}{K(p)} N(p), p \in M^{2}$ are tangent to a fixed oriented plane. In [11] the authors show that the projection of the surface associated to a Laguerre minimal surface is biharmonic.

In [10], the authors present a way to parameterize hypersurfaces as congruence of spheres in which an envelope is contained in a hyperplane. Using this parametrization, they present a generalization of the surfaces of the spherical type (Laguerre minimal surfaces) studied in [8], namely the Weingarten hypersurfaces of the spherical type, i.e. the oriented hypersurfaces of the Euclidean space $M^{n} \subset \mathbb{R}^{n+1}$ satisfying a Weingarten relation of the form

$$
\sum_{r=1}^{n}(-1)^{r+1} r f^{r-1}\left(\begin{array}{l}
n \\
r
\end{array}\right) H_{r}=0,
$$

where $f \in C^{\infty}\left(M^{n} ; \mathbb{R}\right)$ and $H_{r}$ is the rth mean curvature of $M^{n}$. A characterization of these hypersurfaces is obtained using harmonic functions. Also, they classify the Weingarten hypersurfaces of the spherical type of rotation and give explicit examples.

In [12], the authors generalize the parametrization obtained by Machado in [9] in the n-dimensional Euclidean space for hypersurfaces $M^{n}$ in space forms $M^{n+1}(c), c=-1,0,1$. Using this parametrization they generalize the results and definitions of Weingarten hypersurfaces of the spherical type and classify the Weingarten hypersurfaces of the spherical type of rotation in space forms. In [6], the authors study the hypersurfaces of the spherical type degenerated (in short DST-hypersurfaces), these hypersurfaces has the geometric property that the middle spheres pass through the origin of the Euclidean space.

In this paper we study a class of oriented hypersurfaces $M^{n}, n \geq 2$, in Euclidean space. If there exist a hyperplane $\Pi$ such that for all point $p \in M^{n}$ the set of spheres with center in $p+\frac{H_{n-1}(p)}{H_{n}(p)} N(p)$ and radius $h=\frac{H_{n-1}}{H_{n}}$ (middle spheres) are tangent to $\Pi$, where $H_{n}, H_{n-1}$ are the rth mean curvature these hypersurfaces are called hypersurfaces of the spherical type, this class of hypersurfaces includes the surfaces of the spherical type (Laguerre minimal surfaces) studied in [8]. We show that for $n=2$, the classes of surfaces of the spherical type and the Weingarten surfaces of the spherical type coincide, more for larger dimensions this is not true and we give explicit examples. We also introduced a class of hypersurfaces associated to a biharmonic map and we show that the hypersurfaces of the spherical type are associated to a biharmonic map. Finally, we classify the hypersurfaces of the spherical type of rotation.

2. Preliminaries. In this section we give some definitions and results that will serve to show our results.

Let $\Omega$ be an open subset of $\mathbb{R}^{n}$ and $u=\left(u_{1}, u_{2}, \ldots, u_{n}\right) \in \Omega$. Let $M^{n} \subset \mathbb{R}^{n+1}$ be an oriented hypersurface with $n$ distinct principal curvatures $k_{i}$, if $X: \Omega \subset \mathbb{R}^{n} \rightarrow \mathbb{R}^{n+1}, n \geq 2$, is a local parametrization of $M^{n}$ and $N: \Omega \subset \mathbb{R}^{n} \rightarrow \mathbb{R}^{n+1}$ the Gauss map of $M^{n}$. Then if $X$ is parametrized by lines of curvature we have

$$
\begin{gathered}
\left\langle X_{, i}, X_{, j}\right\rangle=\delta_{i j} g_{i i}, \quad 1 \leq i, j \leq n, \\
N_{, i}=-k_{i} X_{, i}, \\
\Gamma_{i j}^{k}=0, \quad \Gamma_{i i}^{i}=\frac{g_{i i, i}}{2 g_{i i}}, \quad \Gamma_{i i}^{j}=-\frac{g_{i i, j}}{2 g_{j j}}, \quad \Gamma_{i j}^{i}=\frac{g_{i i, j}}{2 g_{i i}},
\end{gathered}
$$

where $i, j, k$ are distinct, here the subscript, $i$ denotes the derivative with respect to $u_{i}$.

Definition 2.1. The Mean curvature and the Gauss-Kronecker curvature of $M^{n}$ are given by

$$
H=\frac{1}{n} \sum_{i=1}^{n} k_{i}, K=\prod_{i=1}^{n} k_{i} .
$$

Definition 2.2. The rth-mean curvature $H_{r}$ of $M^{n}$ is defined by

$$
H_{r}=\frac{S_{r}(W)}{\left(\begin{array}{l}
n \\
r
\end{array}\right)}
$$


where, for intergers $0 \leq r \leq n, S_{r}(W)$ is defined by

$$
\begin{aligned}
& S_{0}(W)=1, \\
& S_{r}(W)=\sum_{1 \leq i_{1}<\ldots<i_{r} \leq n} k_{i_{1}} \ldots k_{i_{r}} .
\end{aligned}
$$

We observe that $H=H_{1}$ and $K=H_{n}$.

Definition 2.3. A sphere congruence in $\mathbb{R}^{n+1}$ is a n-parameter family of spheres, with a differentiable radius function, whose centers lie on a hypersurface $M^{n} \subset \mathbb{R}^{n+1}$.

An envelope of a sphere congruence is a hypersurface $M^{n}$ such that each point of the hypersurface $M^{n}$ is tangent to a sphere of the sphere congruence.

If there exist a diffeomorphism $\phi: M^{n} \rightarrow \widetilde{M}^{n}$, a differentiable function $h: M^{n} \rightarrow \mathbb{R}$, unit normal vector fields $N, \widetilde{N}$ of $M^{n}$ and $\widetilde{M^{n}}$ respectively, such that:

a) $q+h(q) N(q)=\phi(q)+h(q) \widetilde{N}(\phi(q)), \forall q \in M^{n}$.

b) The subset $q+h(q) N(q), q \in M^{n}$ is a n-dimensional hypersurface.

We say that $M^{n}$ and $\widetilde{M^{n}}$ are locally associated by a sphere congruence.

Definition 2.4. An oriented surface $M^{2} \subset \mathbb{R}^{3}$ is called surface of the spherical type if the spheres with center in $p+\frac{H(p)}{K(p)} N(p)$ and radius $\frac{H(p)}{K(p)}$ are tangents to a fixed oriented plane, where $N$ is the Gauss map of $M^{2}$.

The following definition was given in [6].

Definition 2.5. An oriented hypersurface $M^{n} \subset \mathbb{R}^{n+1}$ is a hypersurface of the spherical type degenerated (in short DST-hypersurface) if for all $p \in M$ the middle spheres pass through the origin, that is, they satisfy the following relation

$$
2 \Psi H_{n-1}+\Lambda H_{n}=0,
$$

where $\Psi$ and $\Lambda$ are the support function and the quadratic distance function given by $\Psi(p)=\langle p, N(p)\rangle, \Lambda(p)=$ $\langle p, p\rangle, p \in M^{n}$.

The following results were obtained in [10].

Theorem 2.1. An oriented hypersurface $M^{n}$ in $\mathbb{R}^{n+1}, n \geq 2$ is an envelope of sphere congruence, whose other envelope is contained in the hyperplane $\Pi=\left\{\left(x_{1}, x_{2}, \ldots, x_{n+1}\right) \in \mathbb{R}^{n+1}: x_{n+1}=0\right\}$ if, and only if, exist an orthogonal local parametrization of $\Pi, Y: U \subset \mathbb{R}^{n} \rightarrow \Pi$ and a differentiable function $h: U \subset \mathbb{R}^{n} \rightarrow \mathbb{R}$, such that $X: U \subset \mathbb{R}^{n} \rightarrow M^{n}$, given by

$$
X(u)=Y(u)-\frac{2 h(u)}{S}\left[\sum_{j=1}^{n} \frac{h_{, j}}{L_{j j}} Y_{, j}-e_{n+1}\right]
$$

is a parametrization of $M^{n}$, with $e_{n+1}=(0,0, \ldots, 0,1), L_{i j}=\left\langle Y_{, i}, Y_{, j}\right\rangle 1 \leq i, j \leq n$ and

$$
S=\sum_{j=1}^{n} \frac{\left(h_{, j}\right)^{2}}{L_{j j}}+1 \text {. }
$$

Moreover, the Gauss map is given by

$$
N(u)=e_{n+1}+\frac{2}{S}\left[\sum_{j=1}^{n} \frac{h_{, j}}{L_{j j}} Y_{, j}-e_{n+1}\right],
$$

and the Weingarten matrix is given by

$$
W=2 V(S I-2 h V)^{-1}
$$

where the matrix $V=\left(V_{i j}\right)$ is given by

$$
V_{i j}=\frac{1}{L_{j j}}\left(h_{, i j}-\sum_{l=1}^{n} \widetilde{\Gamma}_{i j}^{l} h_{, l}\right), 1 \leq i, j \leq n,
$$


where $\widetilde{\Gamma}_{k i}^{l}$ are the Christoffel symbols of the metric $L_{i j}$.

The regularity condition of $X$ is given by

$$
P=\operatorname{det}(S I-2 h V) \neq 0 .
$$

Corollary 2.1. With the conditions of the Theorem 2.1, we have that the first, second and third fundamental forms are given by

$$
\begin{aligned}
I & =L-\frac{2 h}{S}\left((V L)^{T}+V L\right)+\left(\frac{2 h}{S}\right)^{2} V L V^{T}, \\
I I & =-\frac{2}{S}(V L)^{T}+\frac{4 h}{S^{2}} V L V^{T}, \\
I I I & =\frac{4}{S^{2}} V L V^{T},
\end{aligned}
$$

where $L$ is the matrix of the metric $\left(L_{i j}\right)$ and $T$ denotes the transpose.

Remark 2.1. Let $X: U \subset \mathbb{R}^{n} \rightarrow M^{n} \subset \mathbb{R}^{n+1}$ be a parametrization of the hypersurface $M^{n}$ given by (2.4). Then from (2.7), the principal curvatures are given by (see [10])

$$
k_{i}=\frac{2 V_{i i}}{2 h V_{i i}-S}, \quad 1 \leq i \leq n
$$

where $V_{i i}$ are the eigenvalues of the matrix $V$.

The following results obtained in [10], characterize the Weingarten hypersurfaces of the spherical type and the hypersurfaces of rotation in $\mathbb{R}^{n+1}$, respectively.

Theorem 2.2. Let $M^{n} \subset \mathbb{R}^{n+1} n \geq 2$, be a hypersurface as in Theorem 2.1. $M^{n}$ is a Weingarten hypersurface of the spherical type if, and only if, $\operatorname{tr}(V)=0$.

Theorem 2.3. Let $Y: U \rightarrow \Pi$ be a parametrization of the hyperplane $\Pi$ given by $Y(u)=(u, 0)$, $u \in U, h: U \rightarrow \mathbb{R}$ a differentiable function and $X: U \rightarrow \mathbb{R}^{n+1}$ the immersion given by (2.4) with Gauss map $N$ given by (2.6). Under these conditions $X(U)$ is a hypersurface of rotation if, and only if, $h$ is a radial function.

3. Hypersurfaces of the spherical type. In this section we will define and characterize the hypersurfaces of the spherical type.

Definition 3.1. An oriented hypersurface $M^{n} \subset \mathbb{R}^{n+1}, n \geq 2$, is called hypersurface of the spherical type, if there exist a hyperplane $\Pi$ such that for all point $p \in M^{n}$ the set of spheres with center in $p+\frac{H_{n-1}(p)}{H_{n}(p)} N(p)$ and radius $h=\frac{H_{n-1}}{H_{n}}$ are tangent to $\Pi$, where $H_{n}, H_{n-1}$ are the rth mean curvature.

The following result characterizes the hypersurfaces of the spherical type.

Theorem 3.1. Let $M^{n} \subset \mathbb{R}^{n+1} n \geq 2$, be a hypersurface as in Theorem 2.1.

$M^{n}$ is a hypersurface of the spherical type if, and only if, $\operatorname{tr}\left(V^{-1}\right)=0$.

Proof: Let $V_{i i}$ be the eigenvalues of the matrix $V$ and $k_{i}$ the principal curvatures of $M^{n}, 1 \leq i \leq n$. By equation (2.10), we get

$$
V_{i i}=\frac{S k_{i}}{2 h k_{i}-2}
$$

Thus, the trace of $V^{-1}$ is given by

$$
\begin{aligned}
\operatorname{tr}\left(V^{-1}\right) & =\frac{1}{V_{11}}+\frac{1}{V_{22}}+\ldots+\frac{1}{V_{n n}} \\
& =\frac{2 h k_{1}-2}{S k_{1}}+\frac{2 h k_{2}-2}{S k_{2}}+\ldots+\frac{2 h k_{n}-2}{S k_{n}} \\
& =\frac{\left(2 h k_{1}-2\right) k_{2} \ldots k_{n}+\ldots+\left(2 h k_{n}-2\right) k_{1} k_{2} \ldots k_{n-1}}{S\left(k_{1} k_{2} \ldots k_{n}\right)} \\
& =\frac{2 n h H_{n}-2\left(k_{2} \ldots k_{n}+\ldots+k_{1} k_{2} \ldots k_{n-1}\right)}{S H_{n}} \\
& =\frac{2 n}{S}\left(h-\frac{H_{n-1}}{H_{n}}\right) .
\end{aligned}
$$


From definition 3.1, it follows the result.

Corollary 3.1. Let $M^{2} \subset \mathbb{R}^{3}$, be a surface as in Theorem 2.1. Then $X$ is a surface of the spherical type if, and only if, $X$ is a Weingarten surface of the spherical type.

Proof: Observe that as $V$ is a matrix the order 2, then $\operatorname{tr}(V)=0$ if, and only if, $\operatorname{tr}\left(V^{-1}\right)=0$. Therefore, from Theorems 2.2 and 3.1, we obtain the result.

Remark 3.1. From corollary 3.1, we obtain that for $n=2$, the classes of surfaces of the spherical type and the Weingarten surfaces of the spherical type coincide, more for larger dimensions this is not true, as we can see in the following example.

Example 3.1. Consider the function $h: \mathbb{R}^{n} \rightarrow \mathbb{R}$, given by

$$
h\left(u_{1}, \ldots, u_{n}\right)=\sum_{i=1}^{n} a_{i} u_{i}^{2}+b_{i} u_{i}+c_{i}, \text { such that } \sum_{i=1}^{n} \frac{1}{a_{i}}=0 \text { and } \sum_{i=1}^{n} a_{i} \neq 0,
$$

where $a_{i} \neq 0, b_{i}$ and $c_{i}, 1 \leq i \leq n$, are real constants.

Considering $Y(u)=(u, 0)$, we obtain from (2.4) that

$$
X(u)=(u, 0)-\frac{2 \sum_{i=1}^{n} a_{i} u_{i}^{2}+b_{i} u_{i}+c_{i}}{1+\sum_{i=1}^{n}\left(2 a_{i} u_{i}+b_{i}\right)^{2}}\left(2 a_{1} u_{1}+b_{1}, 2 a_{2} u_{2}+b_{2}, \cdots, 2 a_{n} u_{n}+b_{n},-1\right),
$$

is a hypersurface of the spherical type that is not a Weingarten hypersurface of the spherical type.

In fact, since $h_{, i}=2 a_{i} u_{i}+b_{i}, h_{, i j}=2 a_{i} \delta_{i j}$ and $L_{i j}=\delta_{i j}$, from (2.8) we get that the matrix $V=\left(V_{i j}\right)=$ $\left(h_{, i j}\right)$ is diagonal.

On the other hand,

$\operatorname{tr}\left(V^{-1}\right)=\sum_{i=1}^{n} \frac{1}{2 a_{i}}=0$ and $\operatorname{tr}(V)=\sum_{i=1}^{n} 2 a_{i} \neq 0$, from theorems 2.2 and 3.1, it follows the result.

Remark 3.2. For $n=2$, considering $Y=(g(u), 0)$ in theorem 2.1, where $g(u)$ is a holomorphic function, we obtain that the surface of the spherical type is locally parametrized by

$$
X(u)=(g, 0)-\frac{2 h}{S}\left(\frac{g^{\prime} \cdot \nabla h}{\left|g^{\prime}\right|^{2}},-1\right) .
$$

Moreover, this representation provides examples of Laguerre minimal surfaces.

In the following result we present a class of hypersurfaces that are hypersurfaces of the spherical type and Weingarten hypersurfaces of the spherical type.

Proposition 3.1. Let $Y: \mathbb{R}^{n} \rightarrow \Pi \subset \mathbb{R}^{n+1}, n=2 k$, be a parametrization of hyperplane $\Pi$ given by $Y(u)=\left(g_{1}\left(z_{1}\right), g_{2}\left(z_{2}\right), \ldots, g_{k}\left(z_{k}\right), 0\right)$, where $g_{r}: U_{r} \subset \mathbb{C} \rightarrow \mathbb{C}$ are holomorphic functions, $h^{r}: U_{r} \subset \mathbb{C} \rightarrow \mathbb{R}$ are differentiable functions, $U_{r}$ open set, $u=\left(z_{1}, \ldots, z_{r}, \ldots, z_{k}\right), z_{r}=\left(u_{2 r-1}, u_{2 r}\right)$, $1 \leq r \leq k$.

If $h: U \subset \mathbb{R}^{n} \rightarrow \mathbb{R}$ is given by

$$
h(u)=\sum_{r=1}^{k} h^{r}\left(z_{r}\right)
$$

such that $h^{r}$ satisfy

$$
h_{, 2 r-12 r}^{r}-\widetilde{\Gamma}_{2 r-12 r}^{2 r} h_{, 2 r}^{r}-\widetilde{\Gamma}_{2 r-12 r}^{2 r-1} h_{, 2 r-1}^{r}=0 .
$$

Then $X$ given by (2.4) is a hypersurface parametrized by lines of curvature. Moreover, $X$ is a hypersurface of the spherical type if, and only if, $h^{r}$ is a harmonic function.

Proof: Differentiating $Y$ with relation to $u_{2 r-1}$ and $u_{2 r}$, we have

$$
\begin{aligned}
Y_{, 2 r-1} & =\left(0,0, \ldots, g_{r}^{\prime}, \ldots, 0\right), \\
Y_{, 2 r} & =\left(0,0, \ldots, i g_{r}^{\prime}, \ldots, 0\right) .
\end{aligned}
$$


Thus, $L_{i j}=\left\langle Y_{, i}, Y_{, j}\right\rangle=0,1 \leq i \neq j \leq 2 k$.

Putting $L_{i i}=L_{i}$, for $1 \leq r \leq k$, we get

$$
L_{2 r}=L_{2 r-1}=\left\|g_{r}^{\prime}\right\|^{2} .
$$

From (2.3) we obtain

$$
\begin{aligned}
\widetilde{\Gamma}_{2 r-12 r}^{2 r-1} & =\frac{L_{2 r-1,2 r}}{2 L_{2 r-1}}=\frac{\left\|g_{r}^{\prime}\right\|_{, 2 r}^{2}}{2\left\|g_{r}^{\prime}\right\|^{2}}=\frac{\left\langle i g_{r}^{\prime \prime}, g_{r}^{\prime}\right\rangle}{\left\|g_{r}^{\prime}\right\|^{2}}, \\
\widetilde{\Gamma}_{2 r-12 r-1}^{2 r} & =\frac{-L_{2 r-1,2 r}}{2 L_{2 r}}=-\frac{\left\langle i g_{r}^{\prime \prime}, g_{r}^{\prime}\right\rangle}{\left\|g_{r}^{\prime}\right\|^{2}}, \\
\widetilde{\Gamma}_{2 r 2 r-1}^{2 r} & =\frac{L_{2 r, 2 r-1}}{2 L_{2 r}}=\frac{\left\langle g_{r}^{\prime \prime}, g_{r}^{\prime}\right\rangle}{\left\|g_{r}^{\prime}\right\|^{2}}, \\
\widetilde{\Gamma}_{2 r 2 r}^{2 r-1} & =-\frac{L_{2 r, 2 r-1}}{2 L_{2 r-1}}=-\frac{\left\langle g_{r}^{\prime \prime}, g_{r}^{\prime}\right\rangle}{\left\|g_{r}^{\prime}\right\|^{2}}, \\
\widetilde{\Gamma}_{2 r-12 r-1}^{2 r-1} & =\frac{L_{2 r-1,2 r-1}}{2 L_{2 r-1}}=\frac{\left\langle g_{r}^{\prime \prime}, g_{r}^{\prime}\right\rangle}{\left\|g_{r}^{\prime}\right\|^{2}}, \\
\widetilde{\Gamma}_{2 r 2 r}^{2 r} & =\frac{L_{2 r, 2 r}}{2 L_{2 r}}=\frac{\left\langle i g_{r}^{\prime \prime}, g_{r}^{\prime}\right\rangle}{\left\|g_{r}^{\prime}\right\|^{2}},
\end{aligned}
$$

and $\widetilde{\Gamma}_{i j}^{l}=0$, for $i, j$ and $l$ distinct, $1 \leq i, j, l \leq 2 k$.

Using (2.8) and (3.2) we get that for $1 \leq r \leq k$,

$$
\begin{aligned}
V_{2 r-12 r} & =\frac{1}{L_{2 r}}\left(h_{, 2 r-12 r}-\widetilde{\Gamma}_{2 r-12 r}^{2 r-1} h_{, 2 r-1}-\widetilde{\Gamma}_{2 r-12 r}^{2 r} h_{, 2 r}\right) \\
& =\frac{1}{\left\|g_{r}^{\prime}\right\|^{2}}\left(h_{, 2 r-12 r}^{r}-\frac{\left\langle i g_{r}^{\prime \prime}, g_{r}^{\prime}\right\rangle}{\left\|g_{r}^{\prime}\right\|^{2}} h_{, 2 r-1}^{r}-\frac{\left\langle g_{r}^{\prime \prime}, g_{r}^{\prime}\right\rangle}{\left\|g_{r}^{\prime}\right\|^{2}} h_{, 2 r}^{r}\right)=V_{2 r 2 r-1} .
\end{aligned}
$$

From (3.1) it follows that

$$
V_{2 r-12 r}=0 .
$$

On the other hand

$$
\begin{aligned}
V_{2 r-1 j} & =V_{j 2 r-1}=0, \text { se } j \neq 2 r-1,2 r, \\
V_{2 r j} & =V_{j 2 r}=0, \text { se } j \neq 2 r-1,2 r .
\end{aligned}
$$

From (3.4), (3.5) and the corollary 2 in [10] we obtain that $X$ is parametrized by lines of curvature.

Also,

$$
\begin{aligned}
V_{2 r-12 r-1} & =\frac{1}{L_{2 r-1}}\left(h_{, 2 r-12 r-1}-\widetilde{\Gamma}_{2 r-12 r-1}^{2 r-1} h_{, 2 r-1}-\widetilde{\Gamma}_{2 r-12 r-1}^{2 r} h_{, 2 r}\right) \\
& =\frac{1}{\left\|g_{r}^{\prime}\right\|^{2}}\left(h_{, 2 r-12 r-1}^{r}-\frac{\left\langle g_{r}^{\prime \prime}, g_{r}^{\prime}\right\rangle}{\left\|g_{r}^{\prime}\right\|^{2}} h_{, 2 r-1}^{r}+\frac{\left\langle g_{r}^{\prime}, g_{r}^{\prime}\right\rangle}{\left\|g_{r}^{\prime}\right\|^{2}} h_{, 2 r}^{r}\right)
\end{aligned}
$$

and

$$
\begin{aligned}
V_{2 r 2 r} & =\frac{1}{\left\|g_{r}^{\prime}\right\|^{2}}\left(h_{, 2 r 2 r}-\widetilde{\Gamma}_{2 r 2 r}^{2 r-1} h_{, 2 r-1}-\widetilde{\Gamma}_{2 r 2 r}^{2 r} h_{, 2 r}\right) \\
& =\frac{1}{\left\|g_{r}^{\prime}\right\|^{2}}\left(h_{, 2 r 2 r}^{r}+\frac{\left\langle g_{r}^{\prime \prime}, g_{r}^{\prime}\right\rangle}{\left\|g_{r}^{\prime}\right\|^{2}} h_{, 2 r-1}^{r}-\frac{\left\langle i g_{r}^{\prime \prime}, g_{r}^{\prime}\right\rangle}{\left\|g_{r}^{\prime}\right\|^{2}} h_{, 2 r}^{r}\right) .
\end{aligned}
$$

Therefore

$$
\sum_{r=1}^{k}\left(V_{2 r-12 r-1}+V_{2 r 2 r}\right)=\sum_{r=1}^{k} \frac{1}{\left\|g_{r}^{\prime}\right\|^{2}}\left(h_{, 2 r-12 r-1}^{r}+h_{, 2 r 2 r}^{r}\right)=\sum_{r=1}^{k} \frac{\Delta h^{r}}{\left\|g_{r}^{\prime}\right\|^{2}} .
$$

Thus, the diagonal matrix $V$ is given by 


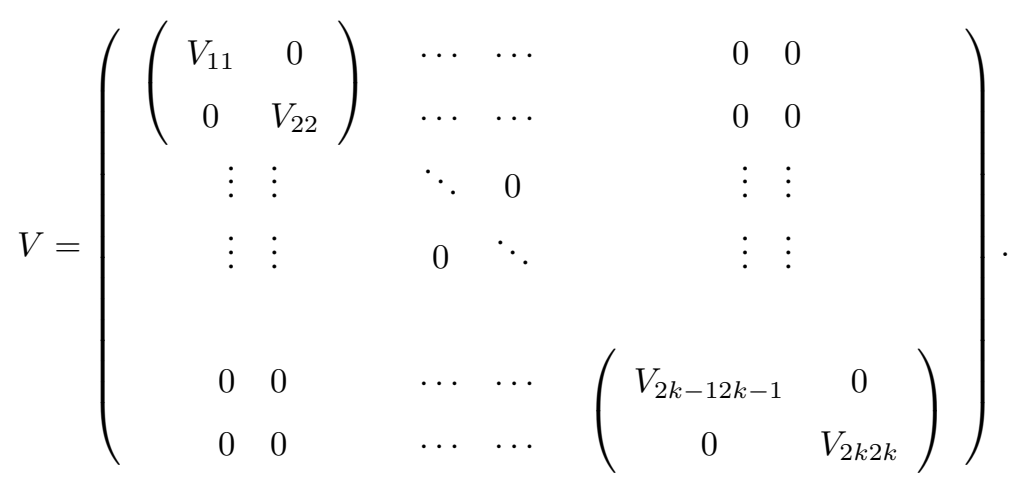

Also, the matrix inverse $V^{-1}$ is given by

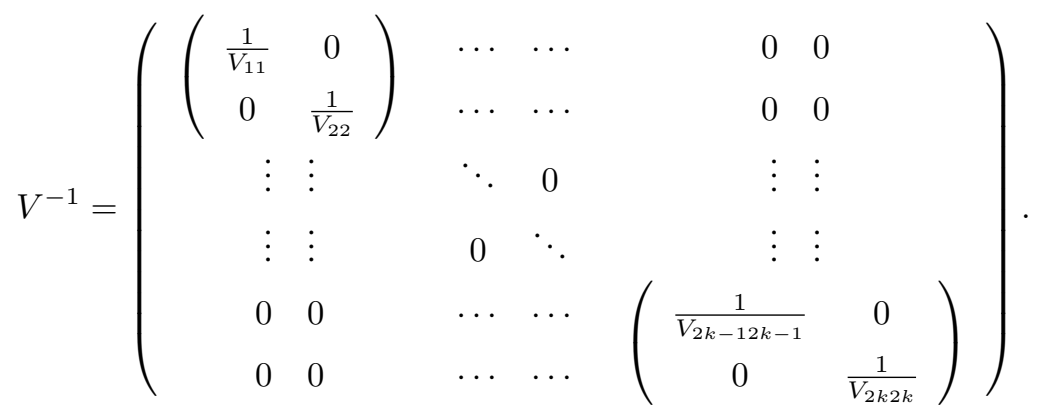

Hence,

$$
\begin{aligned}
\operatorname{tr}\left(V^{-1}\right) & =\sum_{r=1}^{k}\left(\frac{1}{V_{2 r-12 r-1}}+\frac{1}{V_{2 r 2 r}}\right) \\
& =\sum_{r=1}^{k}\left(\frac{V_{2 r-12 r-1}+V_{2 r 2 r}}{V_{2 r-12 r-1} V_{2 r 2 r}}\right) \\
& =\sum_{r=1}^{k}\left(\frac{\Delta h^{r}}{\left\|g_{r}^{\prime}\right\|^{2} V_{2 r-12 r-1} V_{2 r 2 r}}\right) .
\end{aligned}
$$

Therefore,

$$
\operatorname{tr}\left(V^{-1}\right)=0 \text { if, and only if, } \Delta h^{r}=0,
$$

i.e. $h^{r}$ is a harmonic function. From theorem 3.1, it follows the result.

\section{Remark 3.3.}

- From (3.8) we obtain that $\operatorname{tr}(V)=0$ if, and only if, $h^{r}$ is a harmonic function, thus by theorem 2.2, $X$ is a Weingarten hypersurface of the spherical type.

- The hypersurfaces of the spherical type given by theorem 2.1 are not degenerated, in fact, suposse that $M^{n}$ is degenerated, from (2.4), (2.6) and definition 2.5, we obtain that

$$
\Psi=\frac{2}{S}\left(\left\langle Y, \sum_{j=1}^{n} \frac{h_{, j}}{L_{j j}} Y_{, j}\right\rangle-h\right), \Lambda=|Y|^{2}-\frac{4 h}{S}\left(\left\langle Y, \sum_{j=1}^{n} \frac{h_{, j}}{L_{j j}} Y_{, j}\right\rangle-h\right) .
$$

Thus

$$
2 \Psi H_{n-1}+\Lambda H_{n}=0 \Longleftrightarrow Y=0,
$$

which cannot occur because $Y$ is a parameterization of the hyperplane $\Pi$. 
Definition 3.2. An oriented hypersurface $M^{n} \subset \mathbb{R}^{n+1}$ is called a hypersurface associated to a biharmonic map if there exist a hyperplane $\Pi$ such the radius function $h$ determined by $\Pi$, the heigth function $d$ $\left(d(p)\right.$ mesures the distance from $p \in M^{n}$ to $\left.\Pi\right)$ and the rth-mean curvatures $H_{n-1}, H_{n}$ satisfy

$$
\Delta_{I I I}\left(\frac{d}{h} \frac{H_{n-1}}{H_{n}}-d\right)=0
$$

where $\Delta_{I I I}$ is the Laplacian operator with respect to the third fundamental form of $M^{n}$.

Theorem 3.2. Let $M^{n} \subset \mathbb{R}^{n+1} n \geq 2$, be an oriented hypersurface as in theorem $2.1 . M^{n}$ is a hypersurface associated to a biharmonic map if, and only if, $\Delta_{I I I}\left(\operatorname{tr}\left(V^{-1}\right)\right)=0$.

Proof: From (2.10) we have

$$
\frac{1}{k_{i}}=h-\frac{S}{2 V_{i i}}
$$

From definition 2.2 and (3.9) we get

$$
\frac{H_{n-1}}{H_{n}}=\frac{1}{n} \sum_{i=1}^{n} \frac{1}{k_{i}}=h-\frac{S}{2 n} \sum_{i=1}^{n} \frac{1}{V_{i i}}=h-\frac{S}{2 n} \operatorname{tr}\left(V^{-1}\right) .
$$

Also, from (2.4) we obtain that $x_{n+1}=\frac{2 h}{S}=d$, using this expression in the above equation we get

$$
\operatorname{tr}\left(V^{-1}\right)=-n\left(\frac{d}{h} \frac{H_{n-1}}{H_{n}}-d\right) .
$$

Therefore

$$
\Delta_{I I I}\left(\operatorname{tr}\left(V^{-1}\right)\right)=-n \Delta_{I I I}\left(\frac{d}{h} \frac{H_{n-1}}{H_{n}}-d\right) .
$$

Thus, the proof is complete.

Corollary 3.2. Let $M^{n} \subset \mathbb{R}^{n+1} n \geq 2$, be an oriented hypersurface as in theorem 2.1. If $M^{n}$ is a hypersurface of the spherical type. Then $M^{n}$ is a hypersurface associated to a biharmonic map.

Proof: The proof follows from theorem 3.2 and the fact that $\operatorname{tr}\left(V^{-1}\right)=0$.

4. Hypersurfaces of the spherical type of rotation. The following result classifies the hypersurfaces of the spherical type of rotation.

Theorem 4.1. Let $Y: U \subset \mathbb{R}^{n} \rightarrow \Pi$ be a parametrization of the hyperplane $\Pi$ given by $Y(u)=(u, 0)$, $u \in U, h: U \rightarrow \mathbb{R}$ a differentiable function and $X: U \rightarrow \mathbb{R}^{n+1}$ an immersion given by (2.4) with Gauss map $N$ given by (2.6). Under these conditions $X(U)$ is a hypersurface of the spherical type of rotation if, and only if, $h(u)$ is given by

$$
h(u)=\left\{\begin{array}{cl}
C \ln \left(u_{1}^{2}+u_{2}^{2}\right)+D, & \text { if } n=2, \\
\frac{2(n-1) C}{n-2}\left(u_{1}^{2}+\ldots+u_{n}^{2}\right)^{\frac{n-2}{2(n-1)}}+D, & \text { if } n \neq 2,
\end{array}\right.
$$

where $C$ and $D$ are constants, $C>0$.

Proof: If $X(U)$ is a hypersurface of rotation, then from Theorem $2.3 h(u)$ is a radial function, i.e. $h(u)=J(t)$, where $u=\left(u_{1}, \ldots, u_{n}\right)$ and $t=|u|^{2}$.

Therefore,

$$
h_{, i}=2 J^{\prime}(t) u_{i}, h_{, i j}=4 J^{\prime \prime}(t) u_{i} u_{j}+2 J^{\prime}(t) \delta_{i j}, 1 \leq i, j \leq n .
$$

Thus, the matrix $V$ is given by

$$
V=\left(h_{, i j}\right)
$$

is a simetric matrix.

After straightforward calculations we obtain that the elements on the main diagonal $\Delta_{i i}$ of the inverse matrix $V^{-1}$ are given by

$$
\Delta_{i i}=\frac{J^{\prime}(t)+2\left(u_{1}^{2}+u_{2}^{2}+\cdots+\widehat{u_{i}^{2}}+\cdots+u_{n}^{2}\right) J^{\prime \prime}(t)}{2 J^{\prime}(t)\left(J^{\prime}(t)+2 t J^{\prime \prime}(t)\right)}, i=1,2, \cdots, n,
$$


where ${ }^{-}$means that the term is absent in the expression.

Hence, using (4.2) we get

$$
\operatorname{tr}\left(V^{-1}\right)=0 \Longleftrightarrow \sum_{i=1}^{n} \Delta_{i i}=0 \Longleftrightarrow n J^{\prime}(t)+2(n-1) t J^{\prime \prime}(t)=0 .
$$

Therefore

$$
\frac{J^{\prime \prime}(t)}{J^{\prime}(t)}=-\frac{n}{2(n-1) t}
$$

Integrating the above equation

$$
\ln J^{\prime}(t)=-\frac{n}{2(n-1)} \ln t+A \Longleftrightarrow \ln \left(J^{\prime}(t) t^{\frac{n}{2(n-1)}}\right)=A .
$$

Form (4.4) we obtain

$$
J^{\prime}(t)=C t^{-\frac{n}{2(n-1)}}, C=e^{A}
$$

Now, for $n=2$ integrating (4.5) we obtain

$$
J(t)=C \ln t+D
$$

Finally, for $n \neq 2$, integrating (4.5)

$$
J(t)=\frac{2(n-1) C}{n-2} t^{\frac{n-2}{2(n-1)}}+D .
$$

This complete the proof.

Remark 4.1. From Remark 3.1, the results obtained in [10] for Weingarten surfaces of the spherical type of rotation continue to be valid for surfaces of the spherical type of rotation.

5. Conclusions. From the results obtained in this work we can make the following conclusions:

The hypersurfaces of the spherical type generalize the surfaces of the spherical type (Laguerre minimal surfaces), these hypersurfaces has the geometric property that the set of spheres with center in $p+$ $\frac{H_{n-1}(p)}{H_{n}(p)} N(p)$ and radius $h=\frac{H_{n-1}}{H_{n}}$ (middle spheres) are tangent to the hyperplane $\Pi$, we observe that the middle spheres do not pass through the origin of $\mathbb{R}^{n+1}$, therefore, these hypersurfaces are not degenerated. We present a characterization of the hypersurfaces of the spherical type using o trace of a matrix. Also, we show that for $n=2$, the classes of surfaces of the spherical type and the Weingarten surfaces of the spherical type coincide, more for larger dimensions this is not true. We also introduced a class of hypersurfaces associated to a biharmonic map and we show that the hypersurfaces of the spherical type are associated to a biharmonic map. Finally, we classify the hypersurfaces of the spherical type of rotation.

ORCID and License

Carlos M. C. Riveros https: / / orcid.org/0000-0002-1206-7072

Cid D. F. Machado https://orcid.org/0000-0003-4263-9139

This work is licensed under the Creative Commons - Attribution 4.0 International (CC BY 4.0)

\section{References}

[1] Blaschke W. Über die geometrie von Laguerre: I. grundformeln der flächentheorie, Abh. Math. Sem. Univ. Hamburg. 1924; 3:176-194.

[2] Blaschke W. Über die geometrie von Laguerre: II. flächentheorie in ebenenkoordinaten, Abh. Math. Sem. Univ. Hamburg. 1924; 3:195-212.

[3] Blaschke W. Über die geometrie von Laguerre: III. beiträge zur flächentheorie, Abh. Math. Sem. Univ. Hamburg. 1925; 4:1-12.

[4] Blaschke W. Vorlesungen über Differentialgeometrie und geometrische Grundlagen von Einsteins Relativitätstheorie. B. 3, bearbeitet von G. Thomsen, J. Springer, Berlin, 1929. 
[5] Corro AV. Generalized Weingarten surfaces of bryant type in hyperbolic 3-space. Matemática Comtemporânea. 2006; 30:71-89.

[6] Corro AV, Dias D, Riveros CMC. Hypersurfaces of the spherical type degenerated. Selecciones Matemáticas. 2020; 7(2):214221.

[7] Gálvez JA. Martínez, A., Milán, F. Complete linear Weingarten surfaces of bryant type. a plateau problem at infinity. Trans. Amer. Math. Soc. 2004; 356:3405-3428.

[8] Grohs P, Mitra NJ, Pottmann H. Laguerre minimal surfaces, isotropic geometry and linear elasticity. Adv. Comput. Math. 2009; 31(4):391-419.

[9] Machado CDF. Hipersuperfícies Weingarten de tipo esférico, PhD thesis, Universidade de Brasília, 2018.

[10] Machado CDF, Riveros CMC. Weingarten hypersurfaces of the spherical type in Euclidean spaces. Comment. Math. Univ. Carolin. 2020; 61(2):213-236

[11] Muhanna Y, Ali RM. Biharmonic maps and Laguerre minimal surfaces. Abstract and Applied Analysis. 2013; Art. ID 843156, 9 pages.

[12] Reyes EOS, Riveros CMC. Weingarten hypersurfaces of the spherical type in space forms. Serdica Math. J. 2019; 45:259-288. 